\title{
Absolute Determination Method for Trace Quantities of Enantiomer of Glufosinate by $\gamma$-Cyclodextrin Modified Capillary Zone Electrophoresis Combined with Solid-Phase Extraction and On-capillary Concentration
}

\author{
Takayuki ASAMI and Hisanori IMURA ${ }^{\dagger}$ \\ Department of Environmental Sciences, Faculty of Science, Ibaraki University, \\ 2-1-1 Bunkyo, Mito, Ibaraki 310-8512, Japan
}

\begin{abstract}
A highly sensitive and accurate determination method for trace quantities of enantiomers of glufosinate (D,L-GLUF), a phosphorus-containing amino acid-type herbicide, has been studied. The present method is based on the change in the mole ratio of the enantiomeric isomers after spiking of a known amount of an isomer (L-GLUF). The chiral separation and detection were made by $\gamma$-cyclodextrin modified capillary zone electrophoresis $(\gamma$-CD-CZE) with fluorescence detection. Solid-phase extraction of D,L-GLUF with titania was investigated as the pre-separation method to eliminate coexisting materials such as inorganic salts and organic compounds in river water. A separated D,L-GLUF was labeled with dansyl chloride and subjected to the on-capillary concentration using large-volume sample stacking (LVSS) before $\gamma$-CD-CZE. The detection limit of the present method was as low as $2.0 \times 10^{-9} \mathrm{M}$. The present method was successfully applied to a model sample containing $2.0 \times 10^{-7} \mathrm{M}$ D,L-GLUF in river water. It was confirmed that trace quantities of Dand L-GLUF in environmental samples can be accurately determined without any calibration curves and comparison standards.
\end{abstract}

(Received August 21, 2006; Accepted September 29, 2006; Published December 10, 2006)

\section{Introduction}

Nonspecific phosphorus-containing amino acid-type herbicides such as D,L-homoalanine-4-yl(methyl)phosphonic acid (D,LGLUF) have been increasingly used all over the world. D,LGLUF is readily biotransformed to 3-methylphosphinicopropionic acid or 2-methylphosphinicoacetic acid by microorganisms in the soil. ${ }^{1}$ Recently, biochemical studies concerning glufosinate-resistant crops have been also reported. ${ }^{2,3}$ In those studies a racemic mixture was used. On the other hand, it was reported that the half-lives of elimination of D- and L-GLUF in a patient, who ingested the herbicide containing D,L-GLUF, are different from each other, and thus the respective enantiomers have different rates of transfer in the tissues. ${ }^{4}$ Since the physiological properties such as a herbicidal activity are generally different between the enantiomers, a development of a precise, accurate, and simultaneous determination method is required for D- and L-GLUF.

In our recent study, ${ }^{5}$ a chiral separation method for D,LGLUF, which is labeled with dansyl chloride, has been developed by capillary zone electrophoresis using a partially filling technique of $\gamma$-cyclodextrin as a chiral selector $(\gamma-\mathrm{CD}$ CZE). The enantiomers of dansylated D,L-GLUF (Dns-D,LGLUF) were completely separated with $\gamma-C D$ and detected in

† To whom correspondence should be addressed.

E-mail: imura@cacheibm.s.kanazawa-u.ac.jp

Present address: Graduate School of Natural Science \& Technology, Kanazawa University, Kakuma, Kanazawa 920-1192, Japan. the absence of $\gamma$-CD. The absolute determination of D- and LGLUF was achieved based on the principle of enantiomeric isomer dilution (EID) ${ }^{6}$ in which the determination was done by measuring only peak-area ratios of enantiomers without any calibration curves and comparison standards. Moreover, the quantitative recovery and the correction of recovery of D,LGLUF in the dansyl derivatization procedure are not required at all. However, the detection limit of D- and L-GLUF calculated from the electropherogram reported ${ }^{5}$ was relatively high at 12 $\mathrm{mg} / \mathrm{L}\left(6.2 \times 10^{-5} \mathrm{M}\right)$. Since the ambient water quality criteria of $\mathrm{D}$, L-GLUF is $40 \mu \mathrm{g} / \mathrm{L}\left(2.0 \times 10^{-7} \mathrm{M}\right)$, and the reference values for D,L-GLUF residue in crops are $0.05-5$ ppm (in Japan), a more sensitive detection method and preconcentration method has to be developed for the absolute determination of trace quantities of D,L-GLUF.

Therefore, the highly sensitive detection method for D,LGLUF is studied by introducing fluorescence detection and by combining it with a selective solid-phase extraction (SPE) and an on-capillary concentration method. Titanium oxide (titania) is an amphoteric metal oxide and serves as an anion-exchanger at an acidic region and a cation-exchanger at an alkaline region. ${ }^{7,8}$ Titania selectively adsorbs the organic phosphates ${ }^{9}$ and has been used for the selective SPE of phosphoruscontaining amino acid-type herbicides from blood samples under acidic conditions. ${ }^{10}$ The SPE with titania is expected to be an effective pre-separation method for D,L-GLUF in the present work.

Large-volume sample stacking (LVSS) is one of the oncapillary concentration methods in CZE. This is based on the remarkable slowdown of the electrophoretic migrations of ions at the boundary of a low-conductivity sample-matrix into a 
high-conductivity buffer-solution and the elimination of sample matrix by electroosmotic flow (EOF). ${ }^{11}$

In this paper, the SPE behavior of D,L-GLUF in a wide concentration range of $10^{-7}$ to $10^{-4} \mathrm{M}$ is investigated with titania for the pre-separation of trace D,L-GLUF. The LVSS for DnsD,L-GLUF is also studied from the point of view of the efficient concentration and improvement of the resolution in CZE. The sensitive and selective detection method for enantiomers of D,LGLUF is developed by combining the SPE and the LVSS with $\gamma-\mathrm{CD}-\mathrm{CZE}$, and is used for the absolute and chiral determination of trace quantities of D- and L-GLUF. To validate the present method proposed, we applied it to the determination of a trace quantity of D,L-GLUF in the ambient water criteria level $(2.0 \times$ $10^{-7} \mathrm{M}$ ) in a model sample prepared from river water.

\section{Experimental}

\section{Chemicals}

A racemic mixture of D,L-GLUF (97.0\% purity) was purchased from Wako Pure Chemical Industries. L-GLUF was donated by Meiji Seika Kaisha. Stock solutions of D,L-GLUF $\left(1.0 \times 10^{-4} \mathrm{M}\right)$ and L-GLUF $\left(5.0 \times 10^{-5} \mathrm{M}\right)$ were prepared in a $10 \mathrm{mM}$ sodium carbonate solution.

Titania (99.9\% purity, Wako) was of an anatase form. The mean particle size of titania was $5 \mu \mathrm{m}$. Dns-Cl (97.0\% purity, Aldrich) was dissolved in acetone (HPLC grade). Unless otherwise stated, the reagents used were of an analytical reagent grade.

A migration buffer for CZE was a $2.0 \mathrm{mM}$ phosphate buffer solution ( $\mathrm{pH}$ 6.5) which was prepared using sodium dihydrogen phosphate and $\mathrm{NaOH}$. The ionic strength was adjusted to 5.0 $\mathrm{mM}$ with $\mathrm{NaCl}$. A $17 \mathrm{mM} \gamma-\mathrm{CD}(97.0 \%$ purity, Wako) solution was prepared in the phosphate buffer.

Migration buffer solutions were filtrated through a membrane filter with $0.45-\mu \mathrm{m}$ pore size (hydrophilic PTFE, Advantec) immediately before use. Distilled and deionized water was further purified with a MILLI-Q Labo (Millipore) equipment.

\section{Apparatus}

Capillary electrophoresis was performed using a CE900S/FP2025 Plus (JASCO) system equipped with a LC-NETII/ADCPC (JASCO) system at $25^{\circ} \mathrm{C}$. The fluorescence detection wavelength and the excitation wavelength were 557 and 327 $\mathrm{nm}$, respectively. The power supply was operated at a constant voltage of $+30 \mathrm{kV}$ or $-30 \mathrm{kV}$. A fused-silica capillary, $101 \mathrm{~cm}$ in length (69 $\mathrm{cm}$ from the inlet to the detector window) and 50 $\mu \mathrm{m}$ i.d., was used. It was successively flushed with water, 50 v/v\% methanol, water, $2 \mathrm{M} \mathrm{HNO}_{3}$, water, $0.2 \mathrm{M} \mathrm{NaOH}$ solution, water, and then the migration buffer without $\gamma$-CD for 2 min each. The capillary was finally equilibrated with the migration buffer under the electrophoretic conditions at $+30 \mathrm{kV}$ for $30 \mathrm{~min}$. The $\mathrm{pH}$ was measured with a $\mathrm{pH}$ meter (PHM93, Radiometer) equipped with a combination glass electrode at $25^{\circ} \mathrm{C}$. The conductivity was measured with a conductivity meter (CDM92, Radiometer) at $25^{\circ} \mathrm{C}$.

\section{Recommended procedure}

Solid-phase extraction with titania. A 0.1-g portion of titania was added to a 50-ml sample solution of which the $\mathrm{pH}$ was adjusted to 3.0 using trifluoroacetic acid (TFA). The mixture was gently shaken for $10 \mathrm{~min}$ and filtrated through a membrane filter $(0.45 \mu \mathrm{m})$. Titania having adsorbed D,L-GLUF was washed with $10 \mathrm{ml}$ of water and D,L-GLUF was eluted with 5 $\mathrm{ml}$ of $75 \mathrm{mM}$ ammonia solution. The eluate was evaporated to dryness and redissolved in $1 \mathrm{ml}$ of $10 \mathrm{mM}$ sodium carbonate solution.

Large-volume sample stacking/ $\gamma$-CD-CZE. D,L-GLUF was labeled with Dns-Cl according to the procedure given in a previous paper. ${ }^{5}$ A test sample for LVSS was prepared by diluting the Dns-D,L-GLUF solution to 50 times with water and hydrodynamically injected from the injection end into the capillary filled with a migration buffer without $\gamma-\mathrm{CD}$. Almost the whole capillary was filled with the test sample, and then Dns-D,L-GLUF in the capillary was concentrated at the injection end of capillary which was subjected to a negative potential of $-30 \mathrm{kV}$. After LVSS, a migration buffer containing $17 \mathrm{mM} \gamma-\mathrm{CD}$ at $\mathrm{pH} 6.5$ was partially and electrophoretically injected into the capillary at $+30 \mathrm{kV}$ for $15-20 \mathrm{~min}$. The chiral separation and the measurement of the peak-area of Dns-D- and Dns-L-GLUF were performed using the migration buffer without $\gamma-\mathrm{CD}$ at $\mathrm{pH} 6.5$ and $+30 \mathrm{kV}$.

\section{Principle of determination}

Since physicochemical properties of enantiomeric isomers are exactly the same under achiral conditions, the mole ratio of the D-GLUF to the L-GLUF agrees with the ratio of the peak area of the Dns-D-GLUF to that of the Dns-L-GLUF as long as there is a proportional relationship between the fluorescence intensity and the concentration in a capillary in CZE, i.e.,

$$
R_{\mathrm{X}}=\frac{S_{\mathrm{DX}}}{S_{\mathrm{LX}}}=\frac{M_{\mathrm{DX}}}{M_{\mathrm{LX}}}
$$

Here $S_{\mathrm{DX}}$ and $S_{\mathrm{LX}}$ denote the peak area of the Dns-D- and Dns-LGLUF, respectively, and the ratio is written as $R_{\mathrm{X}} ; M_{\mathrm{DX}}$ and $M_{\mathrm{LX}}$ denote the moles of the D- and L-GLUF, respectively.

If a known amount of the L-isomer $\left(M_{\mathrm{LS}}\right)$ is spiked into the sample, the relation of the peak-area ratio to the mole ratio is given by

$$
R_{\mathrm{m}}=\frac{S_{\mathrm{Dm}}}{S_{\mathrm{Lm}}}=\frac{M_{\mathrm{DX}}}{M_{\mathrm{LX}}+M_{\mathrm{LS}}}
$$

where $S_{\mathrm{Dm}}$ and $S_{\mathrm{Lm}}$ denote the peak area of Dns-D- and Dns-LGLUF in the mixture, respectively. From Eqs. (1) and (2), the following equations are derived:

$$
\begin{aligned}
& M_{\mathrm{DX}}=\frac{R_{\mathrm{X}} R_{\mathrm{m}} M_{\mathrm{LS}}}{R_{\mathrm{X}}-R_{\mathrm{m}}}, \\
& M_{\mathrm{LX}}=\frac{R_{\mathrm{m}} M_{\mathrm{LS}}}{R_{\mathrm{X}}-R_{\mathrm{m}}} .
\end{aligned}
$$

These equations demonstrate that the unknown amount of the D- and L-GLUF in the sample can be determined by only measuring $R_{\mathrm{X}}$ and $R_{\mathrm{m}}$ without any calibration curves or comparison standards. Furthermore, a quantitative recovery of D,L-GLUF or the correction of the recovery is not necessary, i.e., quantitative recovery in the pre-separation and concentration, and quantitative yield of labeling of D,L-GLUF are not required at all.

\section{Results and Discussion}

\section{Pre-separation of D,L-GLUF with titania}

The effect of $\mathrm{pH}$ on the adsorption of D,L-GLUF onto titania was studied because D,L-GLUF has different acidic and basic groups such as phosphinate, carboxylate, and amino groups. A 


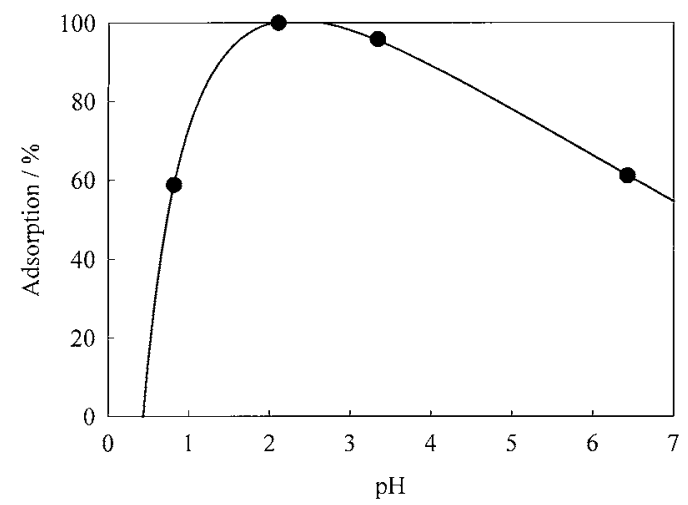

Fig. 1 Effect of $\mathrm{pH}$ on the adsorption of D,L-GLUF onto $0.1 \mathrm{~g}$ titania. Twenty milliliters of $2.5 \times 10^{-4} \mathrm{M}$ D,L-GLUF solution were used and the $\mathrm{pH}$ was adjusted with TFA.

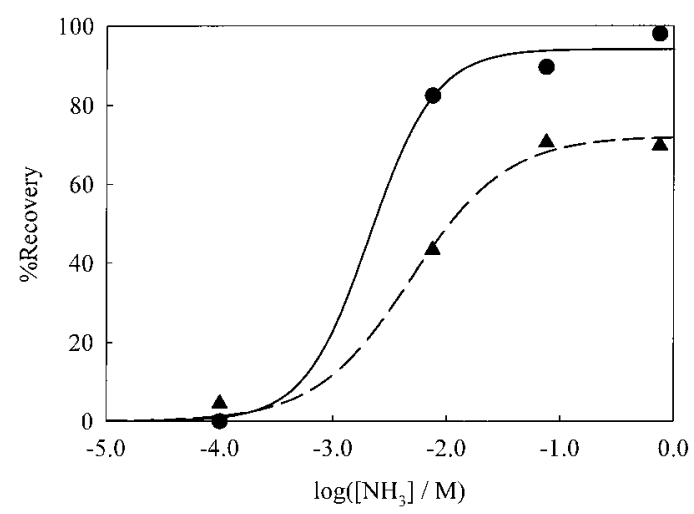

Fig. 2 Effect of the $\mathrm{NH}_{3}$ concentration in the eluent on the recovery of D,L-GLUF. D,L-GLUF was adsorbed from $2.5 \times 10^{-4} \mathrm{M}(\bullet)$ and $4.0 \times 10^{-7} \mathrm{M}(\mathbf{\Lambda})$ solutions onto titania at $\mathrm{pH}$ 3.0. Volume of the eluent $=5 \mathrm{ml}$.

0.1 -g portion of titania was added to $20 \mathrm{ml}$ of $2.5 \times 10^{-4} \mathrm{M}$ D, LGLUF solution at $\mathrm{pH} 1.0-6.5$. TFA was used to adjust the $\mathrm{pH}$ since it does not interfere with the adsorption of D,L-GLUF onto titania and can be readily removed by evaporation. After filtration, D,L-GLUF remaining in the filtrate was determined by the present method based on Eqs. (3) and (4) using L-GLUF as a spike.

Figure 1 shows the $\mathrm{pH}$ dependency of the adsorption of D,LGLUF onto titania. The \%adsorption of D,L-GLUF increases with increase in $\mathrm{pH}$ and reaches the maximum, almost $100 \%$, at $\mathrm{pH}$ 2.0. The isoelectric point of titania is reported to be around $5 .^{12,13}$ Since the $\mathrm{p} K_{\mathrm{a}}$ of phosphinate group of $\mathrm{D}, \mathrm{L}-\mathrm{GLUF}$ is $2.37,{ }^{14}$ phosphinate can be bonded to cationic $\mathrm{Ti}$ at the titania surface at the acidic region, as reported for the phosphate anion. ${ }^{15}$ The adsorption of D,L-GLUF decreased with increase in $\mathrm{pH}$ beyond $\mathrm{pH}$ 3. This phenomenon can be ascribed to the variation of the property of the titania surface which changes from anion exchanger to cation exchanger with increasing $\mathrm{pH} .{ }^{7,8}$ Therefore, the elution of D,L-GLUF from titania should occur at the higher $\mathrm{pH}$ region.

The elution of D,L-GLUF from titania was studied using 0.1 $750 \mathrm{mM}$ ammonia solutions. The titania phase having D,LGLUF was prepared by adsorbing it from $4.0 \times 10^{-7}$ or $2.5 \times$ $10^{-4} \mathrm{M}$ D,L-GLUF solution $(20-50 \mathrm{ml})$ to $0.1 \mathrm{~g}$ titania. The amount of D,L-GLUF eluted was determined by the present method as described above. The effects of concentration and

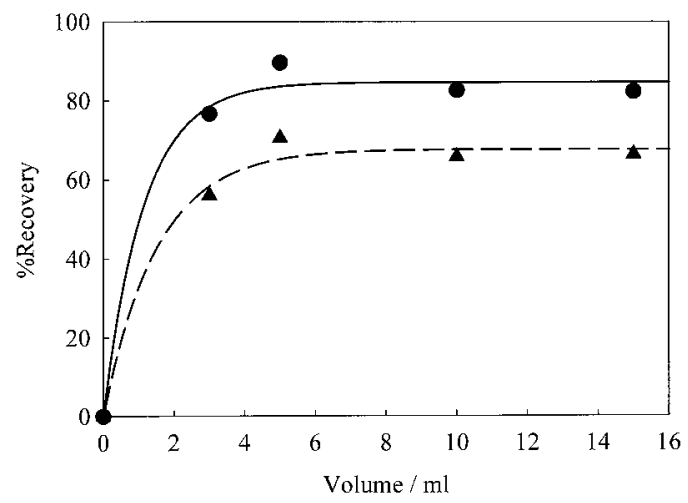

Fig. 3 Effect of the volume of $75 \mathrm{mM} \mathrm{NH}_{3}$ solution as an eluent on the recovery of D,L-GLUF. D,L-GLUF was adsorbed from $2.5 \times 10^{-4}$ $\mathrm{M}(\boldsymbol{\bullet})$ and $4.0 \times 10^{-7} \mathrm{M}(\boldsymbol{\Delta})$ solutions onto titania at $\mathrm{pH} 3.0$.

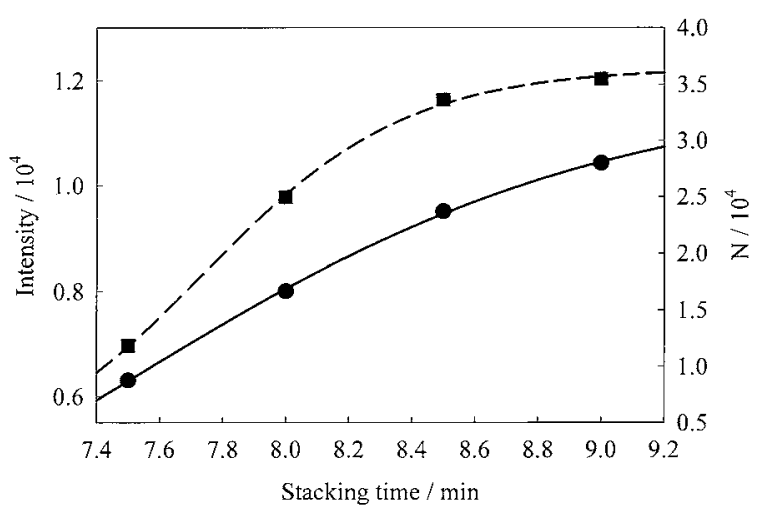

Fig. 4 Effect of the stacking time in LVSS on the intensity (ש) and the theoretical plate number ( ) of Dns-D-GLUF. About $1.5 \mu \mathrm{l}$ of $1.0 \times 10^{-6} \mathrm{M}$ Dns-D,L-GLUF is hydrodynamically injected in the capillary containing the migration buffer without $\gamma$-CD. LVSS is carried out at $-30 \mathrm{kV}$ for $8.0-10.0 \mathrm{~min}$.

volume of ammonia solutions on the elution of D,L-GLUF are shown in Figs. 2 and 3, respectively. The elution of D,L-GLUF increases with increase in the concentration as well as in the volume of the ammonia solution. The optimum conditions were determined to be $5 \mathrm{ml}$ of $75 \mathrm{mM}$ ammonia solution. Therefore, the concentration factor of 4-10 was obtained. Under those conditions, \%recovery was $90 \%$ for $2.5 \times 10^{-4} \mathrm{M}$ D,L-GLUF, compared to $72 \%$ for the concentration as low as $4.0 \times 10^{-7} \mathrm{M}$.

\section{On-capillary concentration of Dns-D,L-GLUF}

A stacking technique is based upon the fact that ions electrophoretically migrate through a low-conductivity samplematrix into a high-conductivity buffer-solution and remarkably slow down at the boundary of the two solutions. ${ }^{11,16}$ The test sample containing Dns-D,L-GLUF was diluted to 50 times upon the addition of water. The conductivity of the test sample reduced to $19.7 \mu \mathrm{S} / \mathrm{cm}$ and was much lower than that of the migration buffer $(400 \mu \mathrm{S} / \mathrm{cm})$. A large volume (around $1.5 \mu \mathrm{l}$ ) of the diluted test sample was injected into the capillary and Dns-D,L-GLUF was concentrated by the electroosmosis at -30 $\mathrm{kV}$ which brings out the elimination of most of the solvent of the test sample out of the capillary.

The stacking time in LVSS affects the concentration efficiency and the zone width of Dns-D,L-GLUF in the capillary. Figure 4 shows the effect of the stacking time on the 
Table 1 Detection limit of D- and L-GLUF

\begin{tabular}{cccc}
\hline \multirow{2}{*}{ Detection } & $\begin{array}{c}\text { Preconcentration } \\
\text { method }\end{array}$ & \multicolumn{2}{c}{ LOD/nM } \\
\cline { 3 - 4 } & & $\mathrm{D}$ & $\mathrm{L}$ \\
\hline UV $^{\mathrm{a}}$ & - & $6.21 \times 10^{4}$ & $5.50 \times 10^{4}$ \\
Fluorescence $^{\mathrm{b}}$ & - & $1.28 \times 10^{4}$ & $1.24 \times 10^{4}$ \\
Fluorescence $^{\mathrm{b}}$ & $\mathrm{LVSS}^{\mathrm{c}}$ & $1.39 \times 10^{2}$ & $1.33 \times 10^{2}$ \\
Fluorescence $^{\mathrm{b}}$ & LVSS + SPE $^{\mathrm{c}}$ & 2.62 & 2.42 \\
\hline
\end{tabular}

a. At $220 \mathrm{~nm}$, from Ref. 5 .

b. At $557 \mathrm{~nm}$ (excitation at $327 \mathrm{~nm}$ ).

c. According to the recommended procedure.

Table 2 Mole ratio and peak-area ratio of D- to L-GLUF

\begin{tabular}{ccccc}
\hline $\begin{array}{c}M_{\mathrm{D}} / 10^{-8} \\
\mathrm{~mol}\end{array}$ & $\begin{array}{c}M_{\mathrm{L}} / 10^{-8} \\
\mathrm{~mol}\end{array}$ & $M_{\mathrm{L}} / M_{\mathrm{D}}$ & $S_{\mathrm{L}} / S_{\mathrm{D}}{ }^{\mathrm{a}}$ & $\begin{array}{c}\text { Deviation, } \\
\%\end{array}$ \\
\hline 1.00 & 1.00 & 1.00 & $0.997 \pm 0.000_{2}$ & 0.3 \\
1.00 & 2.00 & 2.00 & $2.01 \pm 0.04$ & 0.6 \\
1.00 & 3.00 & 3.00 & $2.96 \pm 0.03$ & -1.2 \\
0.500 & 2.52 & 5.04 & $5.02 \pm 0.00_{2}$ & -0.5 \\
0.500 & 5.05 & 10.1 & $10.5 \pm 0.01$ & 3.8 \\
\hline
\end{tabular}

a. Ratio of peak area of D to that of $\mathrm{L}$.

b. $\left(S_{\mathrm{L}} / S_{\mathrm{D}}-M_{\mathrm{L}} / M_{\mathrm{D}}\right) /\left(M_{\mathrm{L}} / M_{\mathrm{D}}\right)$.

peak intensity and the theoretical plate number $(N)$ of Dns-DGLUF as an example. Not only the intensity but also the $N$ value increase with increasing time up to $8.5 \mathrm{~min}$. After 8.5 min, the intensity becomes almost constant, indicating that the conductivity of the concentrated zone containing Dns-D,LGLUF became almost the same as that of the migration buffer. On the other hand, the $N$ value continues to increase since a part of the zone of Dns-D,L-GLUF is eliminated together with the solvent out of the capillary by EOF.

\section{Chiral separation and sensitive detection by LVSS $/ \gamma-C D-C Z E$}

For the chiral separation of Dns-D,L-GLUF after LVSS, $\gamma$ CD-CZE was performed. The partial-filling techniques ${ }^{17,18}$ of $\gamma$ CD were used for detecting Dns-D- and Dns-L-GLUF in the absence of $\gamma-\mathrm{CD}$; otherwise, the peak-area ratio of the $\mathrm{D}-$ to $\mathrm{L}$ isomer does not agree with the mole ratio in the presence of $\gamma$ $\mathrm{CD}$, which will bring about the differences in the migration rate and the electronic absorption spectrum of the D- and L-isomer complex.

The detection limit (LOD), which is defined by 3 times of the standard deviation of noise on the electropherogram, is shown for D-GLUF and for L-GLUF in Table 1. The sensitivities of Dand L-GLUF are effectively enhanced by the fluorescence detection, the LVSS concentration, and their combination with the SPE with titania. The LOD values of D- and L-GLUF attains to $2.62 \times 10^{-9}$ and $2.42 \times 10^{-9} \mathrm{M}$, respectively, which are sufficiently low compared with the value for the environmental water quality criteria, $2.0 \times 10^{-7} \mathrm{M}$. The sensitivities became 23000 times higher than those of simple UV detection method reported previously. ${ }^{5}$

\section{Method validation}

The accuracy and precision of the present absolute determination method depend on the measurement of the peakarea ratio of D- and L-isomers, as found in Eqs. (3) and (4). Therefore, the accuracy of the peak-area ratio measured by $\gamma$ CD-CZE combined with the SPE with titania and the LVSS

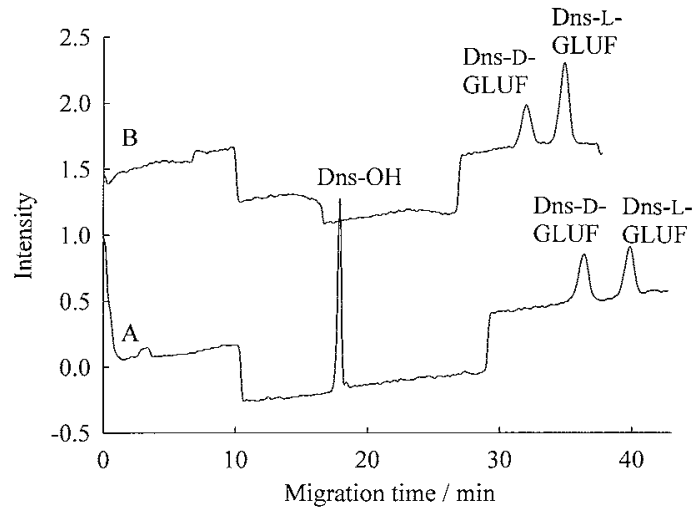

Fig. 5 Electropherograms of Dns-D,L-GLUF prepared from a 50$\mathrm{ml}$ portion of river-water sample containing $2.00 \times 10^{-7} \mathrm{M} \mathrm{D,L}-$ GLUF. CZE conditions, $\mathrm{pH} 6.5,30 \mathrm{kV}$ and $25^{\circ} \mathrm{C}$; LVSS, $-30 \mathrm{kV}$ for $10.5-11.0 \mathrm{~min} ; \gamma$-CD injection, $30 \mathrm{kV}$ for $17.0-18.0 \mathrm{~min}$. (A) Without spike of L-GLUF; (B) after spike of $5.05 \times 10^{-9} \mathrm{~mol} \mathrm{L-GLUF}$.

online-concentration was ascertained using a series of mixtures containing different concentrations of D- and L-GLUF ranging from $1.00 \times 10^{-7}$ to $1.01 \times 10^{-6} \mathrm{M}$. The results of two measurements for each mixture are shown in Table 2. The peak-area ratios obtained are in good agreement with the mole ratios for each mixture except that of $10.1 \times 10^{-7} \mathrm{M}$. Any losses of D,L-GLUF in the pre-separation, derivatization, and preconcentration do not affect the peak-area ratio of Dns-D- to Dns-L-isomer.

The validation of the present method for trace D,L-GLUF was carried out by the recovery test using a river-water sample which was taken at Naka-river (Ibaraki Pref., Japan). The model sample was prepared by adding $0.1 \mathrm{v} / \mathrm{v} \%$ chloroform and $2.00 \times 10^{-7} \mathrm{M}$ D,L-GLUF to the river water, and the mixture was filtrated through a membrane filter with $0.45 \mu \mathrm{m}$ pore size (mixed cellulose ester, Advantec) before use.

According to the recommended procedures, the SPE with titania and then LVSS $/ \gamma$-CD-CZE were carried out after $5.05 \times$ $10^{-9}$ and $10.1 \times 10^{-9} \mathrm{~mol}$ of L-GLUF were spiked to the respective 50-ml portions of the model sample. Since inorganic salts such as $\mathrm{KCl}, \mathrm{K}_{2} \mathrm{SO}_{4}, \mathrm{MgCl}_{2}$, and $\mathrm{MgSO}_{4}$ are not adsorbed onto titania at the neutral $\mathrm{pH}$ region, ${ }^{19}$ those salts on titania could be efficiently eliminated by washing with $10 \mathrm{ml}$ of water. The conductivity of the resulting solution was lowered by 24.7 $\mu \mathrm{S} / \mathrm{cm}$, which was close to the value of $19.7 \mu \mathrm{S} / \mathrm{cm}$ for a test sample prepared using pure water. On the other hand, the recovery of D,L-GLUF in the pre-separation with titania was $71 \%$.

Figure 5 shows the electropherograms obtained from the model sample spiked with $5.05 \times 10^{-9} \mathrm{~mol}$ of L-GLUF (B) and the non-spiked sample (A). A decrease in the baseline (fluorescence intensity) observed at 10 to $28 \mathrm{~min}$ is ascribed to the high concentration of $\gamma-C D$. Since $\gamma-C D$ moves along with the EOF more rapidly than Dns-D,L-GLUF under the given conditions, the $\gamma$-CD zone passes through the band of Dns-D,LGLUF. Dns-D- and Dns-L-GLUF can be separated during the passing of the $\gamma-\mathrm{CD}$ zone and detected in the absence of $\gamma-\mathrm{CD}$. Dns-D- and Dns-L-GLUF are well separated (resolution of $R_{\mathrm{s}}=$ 2.5 ) and sensitively detected. The LODs of D- and L-GLUF were calculated to be $2.17 \times 10^{-9}$ and $2.10 \times 10^{-9} \mathrm{M}$, respectively.

The quantities of D- and L-GLUF in the model sample were calculated by Eqs. (3) and (4) using the peak-area ratio of DnsD- to Dns-L-isomer for $R_{\mathrm{x}}$ and $R_{\mathrm{m}}$ and are summarized in Table 3. 
Table 3 Determination of D- and L-GLUF in a model sample ${ }^{a}$ prepared from river water

\begin{tabular}{|c|c|c|c|c|c|}
\hline $\begin{array}{l}\text { L-GLUF spiked }\left(M_{\mathrm{LS}}\right) / \\
10^{-9} \mathrm{~mol}\end{array}$ & $R_{\mathrm{x}}^{\mathrm{b}}$ & $R_{\mathrm{m}}^{\mathrm{c}}$ & $M_{\mathrm{DX}}^{\mathrm{d} / 10^{-9}} \mathrm{~mol}$ & $M_{\mathrm{LX}} / 10^{-9} \mathrm{~mol}$ & {$[\mathrm{D}, \mathrm{L}-\mathrm{GLUF}] / 10^{-7} \mathrm{M}$} \\
\hline \multirow[t]{2}{*}{5.05} & $\begin{array}{l}0.998 \pm 0.015 \\
(\% \mathrm{RSD}=1.5\end{array}$ & $\begin{array}{c}0.502 \pm 0.013 \\
2.5\end{array}$ & $\begin{array}{c}5.10 \pm 0.25 \\
5.0\end{array}$ & $\begin{array}{c}5.11 \pm 0.24 \\
4.7\end{array}$ & $\begin{array}{c}2.04 \pm 0.07 \\
3.5)^{\mathrm{f}}\end{array}$ \\
\hline & & \multicolumn{2}{|c|}{$(\%$ Deviation $=1.9$} & 2.1 & $2.0)^{\mathrm{g}}$ \\
\hline \multirow[t]{3}{*}{10.1} & $0.998 \pm 0.015$ & $0.324 \pm 0.005$ & $4.85 \pm 0.16$ & $4.86 \pm 0.14$ & $1.94 \pm 0.04$ \\
\hline & $(\% \mathrm{RSD}=1.5$ & 1.4 & 3.3 & 3.0 & $2.2)^{\mathrm{f}}$ \\
\hline & & \multicolumn{2}{|c|}{$(\%$ Deviation $=-2.9$} & -2.7 & $-2.8)^{\mathrm{g}}$ \\
\hline
\end{tabular}

Mean value of $[\mathrm{D}, \mathrm{L}-\mathrm{GLUF}]=(1.99 \pm 0.04) \times 10^{-7} \mathrm{M}, \% \mathrm{RSD}=2.1 \%$, and $\%$ deviation from the amounts taken $=0.5 \%$. a. $50 \mathrm{ml}$ of the sample containing $2.00 \times 10^{-7} \mathrm{M}$ D,L-GLUF was used. The quantity of each isomer was $5.00 \times 10^{-9}$ mol. b. $n=6$. c. $n=3$. d. $M_{\mathrm{DX}}=$ $\left(R_{\mathrm{X}} R_{\mathrm{m}} M_{\mathrm{LS}}\right) /\left(R_{\mathrm{X}}-R_{\mathrm{m}}\right)$. e. $M_{\mathrm{LX}}=\left(R_{\mathrm{m}} M_{\mathrm{LS}}\right) /\left(R_{\mathrm{X}}-R_{\mathrm{m}}\right)$. f. The values in parentheses show relative standard deviations. $\mathrm{g}$. The values in parentheses show deviations from the amounts taken.

The $R_{\mathrm{x}}$ value measured, 0.998 , indicates that D,L-GLUF exists as a 1:1 racemic mixture in this sample. The reproducibility of the measurement of $R_{\mathrm{x}}$ (second column) and $R_{\mathrm{m}}$ (third column) is as good as $1.4-2.5 \%$ as the relative standard deviation (RSD). The RSD values of $M_{\mathrm{DX}}$ and $M_{\mathrm{Lx}}$ somewhat increase to 3.0 $5.0 \%$ because of the propagation of uncertainty derived from Eqs. (3) and (4). The $M_{\mathrm{DX}}$ and $M_{\mathrm{LX}}$ values are well consistent with the quantities taken, $5.00 \times 10^{-9} \mathrm{~mol}$ for each enantiomer within the uncertainty. Since the quantity of L-GLUF spiked has an influence on the uncertainties of $M_{\mathrm{DX}}$ and $M_{\mathrm{LX}}$ through the propagation of uncertainty, there is an optimum mole ratio of the spike to the sample. For example, when $5.0 \times 10^{-10}, 5.0 \times 10^{-9}$, and $1.0 \times 10^{-8} \mathrm{~mol}$ of L-GLUF are spiked to the $1.0 \times 10^{-8} \mathrm{~mol}$ racemic mixture, the RSD value of $M_{\mathrm{DX}}$ or $M_{\mathrm{LX}}$ is calculated to be $21.1,3.6$ and $3.0 \%$, respectively, by assuming that the RSD in the measurement of peak-area of D- and L-isomer is constant as $1 \%$. Table 3 also shows that the difference in the quantity of L-GLUF spiked does not affect the determined values.

The mean value of the total concentration of D,L-GLUF in the model sample of river water is $(1.99 \pm 0.04) \times 10^{-7} \mathrm{M}$ and is in good agreement with the value for that prepared, $2.00 \times 10^{-7} \mathrm{M}$. The deviation of the mean value from the concentration prepared was less than $0.5 \%$, indicating good accuracy of the present absolute determination method. The trace quantity of D,L-GLUF and its enantiomers in an environmental sample can be precisely and accurately determined by the present method.

\section{Conclusions}

The sensitive and reliable determination method of enantiomers of D,L-GLUF was developed. The trace amounts of D- and Lisomer in a river-water sample could be accurately and simultaneously determined without any calibration curves and comparison standards. The traces of D,L-GLUF were separated and concentrated by SPE with titania and further concentrated by the LVSS technique in CZE after dansylation of D,L-GLUF. In all the procedures, the quantitative recovery or the correction of recovery of D,L-GLUF was not required at all because the determination was done by only measuring the ratio of the peakarea of D- to that of L-isomer by $\gamma$-CD-CZE. Therefore, those procedures could be greatly simplified, becoming very easy. The present absolute determination method or this principle can be applied to various optical isomers.

\section{Acknowledgements}

The authors would like to thank Prof. K. Ohashi (Ibaraki
University) and Dr. A. Ohashi (Ibaraki University) for their valuable discussions. The present research was partly supported by the Sasakawa Scientific Research Grant from the Japan Science Society and a Grant-in-Aid for Scientific Research (c) from the Japan Society for the Promotion of Science.

\section{References}

1. C. D. Stalikas and C. N. Konidari, J. Chromatogr., A, 2001, 907, 1.

2. C. Hérouet, D. J. Esdaile, B. A. Mallyon, E. Debruyne, A. Schulz, T. Currier, K. Hendrickx, R.-J. van der Klis, and D. Rouan, Regul. Toxicol. Pharmacol., 2005, 41, 134.

3. E. M. Kishchenko, I. K. Komarnitskii, and N. V. Kuchuk, Cell Biol. Int., 2005, 29, 15.

4. Y. Hori, M. Fujisawa, K. Shimada, M. Sato, M. Honda, and Y. Hirose, J. Chromatogr., B, 2002, 776, 191.

5. T. Asami, H. Imura, A. Ohashi, K. Ohashi, and T. Ishiwata, Anal. Sci., 2005, 21, 31.

6. H. Imura, Y. Oda, K. Ohashi, and H. Kawamura, CITAC '99, Japan Symposium on Practical Realization of Metrology in Chemistry for 21st Century, 1999, Tsukuba, Japan.

7. K. Tani and Y. Suzuki, J. Chromatogr., A, 1996, 722, 129.

8. K. A. Kraus and H. O. Phillips, J. Am. Chem. Soc., 1956, $78,249$.

9. M. Kawahara, H. Nakamura, and T. Nakajima, Anal. Sci., 1989, 5,485 .

10. T. Ishiwata, K. Takai, H. Okada, and K. Ohashi, Jpn. J. Forensic Toxicol., 2004, 22, 1.

11. D. M. Osbourn, D. J. Weiss, and C. E. Lunte, Electrophoresis, 2000, 21, 2768.

12. T. Yazawa, in "A New Phase on Organic/Inorganic Nanocomposite Materials (in Japanese)", ed. Bookers, 2004, NTS, Tokyo, 5.

13. B. P. Nelson, R. Candal, R. M. Corn, and M. A. Anderson, Langmuir, 2000, 16, 6094.

14. T. Asami, H. Imura, T. Ishiwata, and K. Ohashi, Abstract of the 52th Annual Meeting of the Japan Society for Analytical Chemistry, 2003, Sendai, Japan, 177.

15. P. A. Connor and A. J. McQuillan, Langmuir, 1999, 15, 2916.

16. J. L. Beckers and P. Boček, Electrophoresis, 2000, 21, 2747.

17. S. Cherkaoui and J.-L. Veuthey, J. Pharm. Biomed. Anal., 2002, 27, 615.

18. H. Lodén, Y. Hedeland, M. Hedeland, U. Bondesson, and C. Pettersson, J. Chromatogr., A, 2003, 986, 143.

19. C. Labbez, P. Fievet, A. Szymczyk, A. Vidonne, A. Foissy, and J. Pagetti, J. Membr. Sci., 2002, 208, 315. 